\title{
Dynamics of infinite-range ballistic aggregation
}

\author{
Zheming Cheng and Robert Savit \\ Physics Department, University of Michigan, Ann Arbor, Michigan 48109, USA
}

Received 30 June 1987

\begin{abstract}
We calculate the width of the growing interface of ballistic aggregation in the limit in which the range of the sticking interaction between the particles becomes infinite. We derive a scaling form for the width, and we compute the short- and long-time exponents finding $\nu=\frac{3}{4}$ and $\alpha=\frac{1}{2}$. Furthermore, we find that the crossover exponent defining the argument of the scaling function is $\gamma=\frac{1}{2}$. We compare these exact results with computer simulations, finding excellent agreement. We also discuss the relation of these results to those of ordinary finite-range ballistic aggregation. Finally, we present a simple expression for the density of all ballistic aggregation clusters, regardless of the range of the interaction, which agrees with known results and interpolates between the infinite- and finite-range cases.
\end{abstract}

Because of its apparent simplicity and evident applicability to a variety of experimental situations, ballistic aggregation is one of the most commonly studied of the many models of non-equilibrium growth (Vold 1963, Sutherland 1966, Meakin 1985, Bensimon et al 1984, Family and Vicsek 1985, Meakin et al 1986). The model is believed to be applicable to several different kinds of deposition processes including physical vapour deposition and molecular-beam epitaxy. In addition, the growth algorithms defining ballistic aggregation are extremely simple to state and to implement on a computer, and the resulting aggregates display many surprising and intriguing features.

Unfortunately, despite its simplicity, attempts to directly analyse ballistic aggregation have not met with much success. Nevertheless, some analytical progress has been made by studying models related to ballistic aggregation, but which are easier to solve (Meakin et al 1986, Kardar et al 1986, Gelband and Strenski 1985). One such model is an extreme limit of ordinary ballistic aggregation in which the range of the interaction between the particles is taken to infinity. The static properties of this infinite-range ballistic aggregation (IRBA) have been studied recently by Gelband and Strenski (1985), who derived a number of exact results for the density and correlation functions of the aggregate. In this paper, we will discuss the growth dynamics of this model. In particular, we will compute exactly the exponents describing the long-and short-time behaviour of the growing interface.

To begin, let us first review the model. The simplest manifestation of ordinary ballistic aggregation is obtained by considering a two-dimensional square lattice. (The generalisation to higher dimensions will be obvious.) Place a line of $L$ particles (the substrate) along the $x$ axis. For simplicity, we will choose periodic boundary conditions in the $x$ direction. At each time step, randomly choose a value of $x$ and drop a particle in the $y$ direction along the lattice line $x=c$ until it sticks to the aggregate. The simplest sticking rules are that the particle dropped along the line $x=c$ sticks at the lattice site $x=c, y=\max [h(c-1), h(c)+1, h(c+1)]$, where $h(a)$ is the largest value of $y$ at which 
a particle occupies a lattice site in the column $x=a$. This model, with different choices of dimension and substrate, has been discussed by a number of authors (Vold 1963, Sutherland 1966, Meakin 1985, Bensimon et al 1984, Family and Vicsek 1985, Meakin et al 1986).

An interesting modification of this algorithm, which we shall discuss in this paper, is to change the range of the sticking interaction so that the particle dropped in column $x=c$ sticks at $x=c, y=\max [h(c-r), h(c-r+1), \ldots, h(c)+1, h(c+1)$, $h(c+2), \ldots, h(c+r)]$. If $r=L$, we have infinite-range ballistic aggregation.

Let us consider the roughness of the growing interface of IRBA as a function of $L$ and of

$$
\langle h\rangle \equiv \frac{1}{L} \sum_{x} h(x)
$$

the average height of the interface above the substrate. We take, as a measure of the roughness, the standard deviation of the heights of the columns, i.e.

$$
\Delta h \equiv\left(\left\langle h^{2}\right\rangle-\langle h\rangle^{2}\right)^{1 / 2} .
$$

We are particularly interested in the short- and long-time behaviour of $\Delta h$. It is typical in growth models that the behaviour of $\Delta h$ in these limits is determined by two exponents $\nu$ and $\alpha$ such that

$$
\begin{array}{ll}
\Delta h \sim\langle h\rangle^{\prime \prime} & L \rightarrow \infty \text { (short times) } \\
\Delta h \sim L^{\alpha} & \langle h\rangle \rightarrow \infty \text { (long times). }
\end{array}
$$

We will now explicitly verify these scaling relations for IRBA and calculate $\nu$ and $\alpha$. Our calculations assume that the particles in different layers are independently distributed, and that they are randomly distributed within the same layer. Since we are working in the limit of large $L$, these assumptions are quite reasonable. It is clear from the growth algorithm that there is only one particle in each layer which violates these assumptions, and that is the particle which begins a new layer and is therefore constrained to lie directly above a particle in the previous layer. For large $L$, this will have a negligible effect on the distribution.

Let us consider then a cluster formed by the IRBA algorithm with $n$ completed layers. (Note that once a new layer is begun, the IRBA algorithm does not allow any particles to penetrate to previous layers.) Because the particle distribution is random and independent as described above, the quantities $\langle h\rangle$ and $\Delta h$, which are averages taken over a single large cluster, can be computed by averaging over an ensemble of clusters. Let $l$, be the number of particles in layer $i$ for some sample of IRBA, and let $Q\left(l_{i}\right)$ be the probability to have exactly $l$, particles in the $i$ th layer. Then following the simple calculation of Gelband and Strenski (1985) we have

$$
Q\left(l_{1}\right)=\frac{l, L !}{L^{L+1}\left(L-l_{1}\right) !}
$$

and the probability to have the configuration $\left\{l_{1}, l_{2}, \ldots, l_{n}\right\}$ is

$$
T\left(\left\{l_{1}\right\}\right)=\prod_{l=1}^{n} Q\left(l_{l}\right) .
$$


Now consider a given column in an IRBA cluster. Using the assumption that the particles are independently and randomly distributed, the probability that a given column has a height $h$ is

$$
P(h)=\frac{l_{n-h}}{L} \prod_{i=0}^{h-1}\left(1-l_{n-1} / L\right)
$$

so that for a given set $\left\{I_{1}\right\}$, the average height in that cluster is

$$
\bar{h}=\sum_{h} h P(h) \text {. }
$$

To compute $\langle h\rangle$, we must average $(6)$ over all sets $\left\{l_{l}\right\}$ to obtain

$$
\langle h\rangle=\sum_{\left\{l_{i}\right\}} T\left(\left\{l_{i}\right\}\right)\left\{\sum_{h} h P(h)\right\} .
$$

Equations analogous to (7) and (8) apply also to $(\Delta h)^{2}$, as well as to any other quantity composed of independent sums over columns of the cluster.

The sums in (8) can be performed easily by noting that $P(h)$ and $T(\{l\})$ are composed of independent factors, each depending on one $l_{i}$. Because there is no explicit dependence on the column position, the sums over the $l$ are independent of $i$. Thus, after a little algebra, (8) becomes

$$
\langle h\rangle=\sum_{h=0}^{n} h\left(1-\frac{\langle l\rangle}{L}\right)^{n-h+1} \frac{\langle l\rangle}{L}
$$

where

$$
\langle l\rangle=\sum_{l=1}^{L}[l Q(l)]
$$

Similarly, we can derive an expression for $\left\langle h^{2}\right\rangle$ which is

$$
\left\langle h^{2}\right\rangle=\sum_{h=0}^{n} h^{2}\left(1-\frac{\langle l\rangle}{L}\right)^{n-h+1} \frac{\langle l\rangle}{L} .
$$

To proceed, we need to estimate the dependence of $\langle l\rangle$ on $L$. One derivation of this dependence is given in Gelband and Strenski (1985). Here we give a slightly simpler argument: $Q(l)$ is positive, singly peaked and normalised to one. It is therefore clear that

$$
\sum_{i=1}^{L}[l Q(l)]>\frac{1}{2} l_{0}
$$

where $l_{0}$ is the position of the peak of $Q(l)$. Since $Q(l) / Q(l+1)=l(l+1) / L$, the peak is at a position which is of the order of $\sqrt{ } L$. On the other hand,

$$
\begin{aligned}
\langle l\rangle & =\sum_{l=1}^{L} \frac{l^{2} L !}{L^{l+1}(L-l) !}=\frac{L !}{L^{L+1}} \sum_{l=0}^{L-1} \frac{L^{\prime}(L-l)^{2}}{l !} \\
& <\frac{L !}{L^{L+1}} \sum_{i=0}^{x} \frac{L^{\prime}(L-l)^{2}}{l !}=\sqrt{2 \pi L} .
\end{aligned}
$$

Thus $\frac{1}{2} l_{0} \sim \sqrt{ } L<\langle l\rangle<\sqrt{2 \pi L}$ so that $l$ is of the order of $\sqrt{ } L$.

We are now in a position to calculate $\nu$ and $\alpha$. It is straightforward to carry out the sums in (9) and (11), and we find

$$
\langle h\rangle=(n-1)-\frac{L}{\langle l\rangle}\left[1-\left(1-\frac{\langle l\rangle}{L}\right)^{n+1}\right]
$$


and

$$
(\Delta h)^{2}=\left\langle h^{2}\right\rangle-\langle h\rangle^{2}=\frac{L^{2}}{\langle l\rangle^{2}}-\frac{L}{\langle l\rangle}-(2 n-1) \frac{L}{\langle l\rangle}\left(1-\frac{\langle l\rangle}{L}\right)^{n+1}-\frac{L}{\langle l\rangle^{2}}\left(1-\frac{\langle l\rangle}{L}\right)^{2 n+2} \text {. }
$$

Defining

$$
y \equiv(n+1) / \sqrt{ } L
$$

we can write (14) and (15) in the limit of large $L$ and $n$ as

$$
\begin{aligned}
& \langle h\rangle / \sqrt{ } L=y+\mathrm{e}^{-y}-1 \quad(L \text { large }) \\
& (\Delta h)^{2} / L=1-\mathrm{e}^{-2 y}-2 y \mathrm{e}^{-y} \quad(L \text { large). }
\end{aligned}
$$

We may write (17) as

$$
(\Delta h)^{2}=L\{1-\exp [-2 g(\langle h\rangle / \sqrt{ } L)]-2 g(\langle h\rangle / \sqrt{ } L) \exp [-g(\langle h\rangle / \sqrt{ } L)]\}
$$

where $y=g(x)$ is the inverse function of $x=y+\mathrm{e}^{-y}-1$. The asymptotic scaling relations follow immediately:

$$
(\Delta h)^{2}=L F(\langle h\rangle / \sqrt{ } L)
$$

where the scaling function $F$ has the properties

$$
F(x \rightarrow 0) \rightarrow x^{3 / 2} \quad F(x \rightarrow \infty) \rightarrow 1 .
$$

For short times, $y \ll 1$. Using (17), (19) and (20), it is easy to see that for short times $\Delta h \sim\langle h\rangle^{3 / 4} L^{1 / 8}$, so that the short-time exponent $\nu=\frac{3}{4}$. For long times, $y \gg 1$ and $\Delta h \sim L^{1 / 2}$, so that the long-time exponent $\alpha=\frac{1}{2}$. It is common to define a crossover exponent $\gamma$ which controls the way in which $\langle h\rangle$ scales with $L$. This can be read off from (19), which tells us that $\gamma=\frac{1}{2}$.

In figure 1 , we compare the results of computer simulations of IRBA with our calculation (19). In this figure, we have plotted $\ln (\Delta h / \sqrt{ } L)$ against $\ln (\langle h\rangle / \sqrt{ } L)$. The

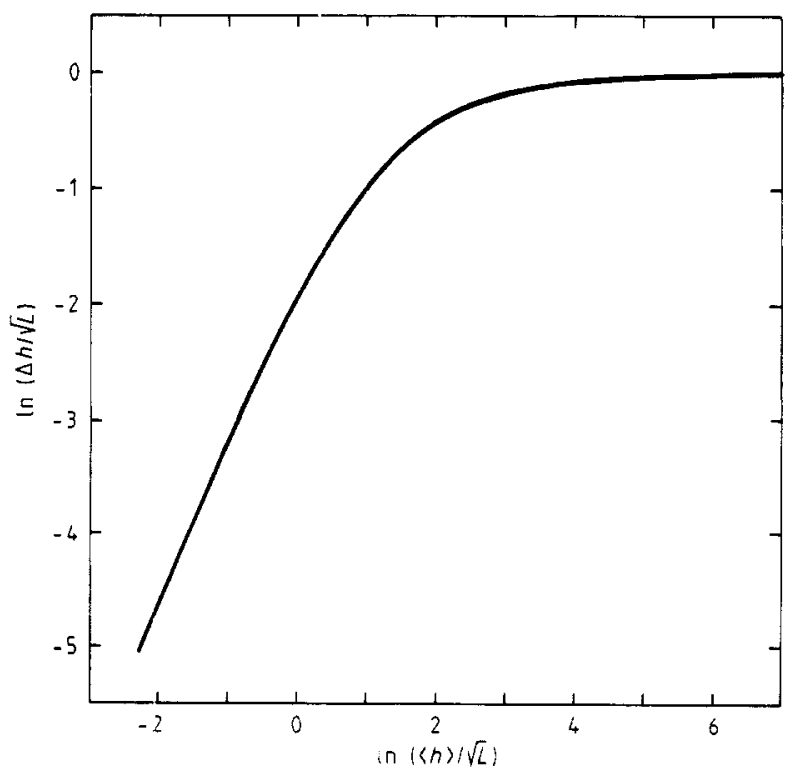

Figure 1. The scaling function equation (19). See text for detailed explanation. 
full curve is equation (19). If our scaling results are correct, all the data for different values of $L$ should lie along the same curve, given by (19). We have performed simulations of IRBA with $L=100000,50000,25000,12500,6250$ and 3125. All the data from these simulations fall directly on the curve in figure 1 to within the resolution of the graph. For somewhat smaller values of $\ln (\langle h\rangle / \checkmark L)$ than shown in figure 1 , the data for different values of $L$ deviate from each other due to initial transient effects. These transients disappear after one complete layer is formed on the substrate. Because particles are deposited randomly along the substrate, it is not difficult to see that in the transient region the slope of the $\ln (\Delta h)$ against $\ln (\langle h\rangle)$ curve is $\frac{1}{2}$, independent of $L$.

It is interesting to compare our results for $\nu, \alpha$ and $\gamma$ to those obtained in ordinary short-range ballistic aggregation. In the short-range case (in any dimension) simple scaling arguments can be used (Meakin et al 1986) which suggests that $\gamma=\alpha / \nu$ and that $2 \nu=\alpha(\nu+1)$. The generally accepted values of $\nu, \alpha$ and $\gamma$ for short-range ballistic aggregation in two dimensions, as determined using both numerical and analytical methods, are $\nu=\frac{1}{3}, \alpha=\frac{1}{2}$ and $\gamma=\frac{3}{2}$. In three dimensions the situation is much less clear, with computer simulations indicating $\nu \approx \frac{1}{4}$ and $\alpha \approx \frac{1}{3}$, while various other arguments give $\nu \approx 0, \alpha \approx 0$ or $\nu \approx \frac{1}{3}, \alpha \approx \frac{1}{2}$.

Our results are in marked contrast to the behaviour found in ordinary short-range ballistic aggregation. In our case, because of the explicit long-range interaction, the simple scaling arguments used in the short-range case do not apply. For instance, even in the short-time regime, $\Delta h$ depends on $L$ in IRBA. Furthermore, the crossover behaviour in IRBA is qualitatively different than in the two-dimensional short-range case. For IRBA the long-time behaviour sets in when the height is only of the order of the square root of the substrate size, which is much sooner than in the twodimensional short-range case. In addition, whether or not the exponent relations presented in the last paragraph always apply in the short-range case, they clearly are not satisfied in IRBA as our exact values of $\alpha, \nu$ and $\gamma$ indicate $\dagger$.

It would be tempting to try to identify IRBA with the $d \rightarrow \infty$ limit of short-range ballistic aggregation. However, this is probably incorrect. The $d \rightarrow \infty$ limit of shortrange ballistic aggregation is obtained by considering short-range interactions, studying the problem for large $L$ and/or $\langle h\rangle$, and then letting the number of nearest neighbours increase. In IRBA $r$, the range of the interaction, is set equal to $L$ from the beginning, and it seems unlikely that the limits $(L,\langle h\rangle) \rightarrow \infty$ and $r \rightarrow L$ commute. Moreover, if IRBA were the $d \rightarrow \infty$ limit of short-range ballistic aggregation, we might expect the short-range exponent relations to be valid, which they are not.

Despite these differences, IRBA is clearly interesting in its own right. Moreover, one can consider a class of theories with interactions which interpolate between IRBA and short-range ballistic aggregation and can thereby relate the two. To illustrate this, we show how such a range of models can elucidate some of the static properties of ballistic aggregation. Consider ballistic aggregation grown on a linear substrate of length $L$, such that $r$, the range of the sticking interaction, is $r \sim L^{p}$. Let us ask, what is $\langle l\rangle$ in this case? Roughly, a layer will be complete when there is at least one particle in each region of length $r$ that is directly above a particle on the previous layer. There are $L / r$ such regions, so $\langle l\rangle \sim(L / r) \times($ the number of particles per region of length $r$ such that at least one is directly above a particle on the previous layer $) \equiv(L / r) t$. Now suppose we drop a particle in a given region of length $r$ which is the first particle in

\footnotetext{
$\div$ We note, however, that $\alpha=\frac{1}{2}$ both in IRBA and two-dimensional finite-range ballistic aggregation. The significance of this intriguing correspondence is not clear to us.
} 
that region in that layer. If we continue to drop particles randomly in the region of length $r$, it is not difficult to see that we must drop of the order of $r^{1 / 2}$ particles to have a finite probability that a particle land above one already in that layer. Thus $t \sim r^{1 / 2}$ and $\langle l\rangle \sim L r^{-1 / 2} \sim L^{1-p / 2}$. The density of ballistic aggregation thus depends on $L$ as $L^{-p / 2}$. This agrees with and interpolates between the conclusions of Ball and Witten (1984) for $p=0$, and with Gelband and Strenski (1985) for $p=1$. It should be possible to construct similar arguments for the dynamic properties of ballistic aggregation.

In this paper we have described the dynamic properties of infinite-range ballistic aggregation. We have exactly calculated the long- and short-time scaling exponents as well as the crossover exponent, and have computed the scaling function. As we have shown, the asymptotic behaviour of IRBA is qualitatively different from that of short-range ballistic aggregation. Nevertheless, it is possible to relate the finite- and infinite-range theories by defining a class of systems with interactions which fall off like a power of the size of the substrate. Using this interpolation, we were able to deduce the form of the density in all these cases. Such a class of interpolating theories should be equally useful for studying the dynamics of ballistic aggregation, and will shed light on the relation of IRBA to the short-range problem.

\section{Acknowledgment}

This work was supported by the US Department of Energy under grant no DE-FG0285ER45189.

\section{References}

Ball R and Witten T 1984 Phys. Rev. A 292966

Bensimon D, Shraiman B and Liang S 1984 Phys. Lett. 102A 238

Family F and Vicsek T 1985 J. Phy.s. A: Math. Gen. 18 L75

Gelband P and Strenski P 1985 J. Phys. A: Marh. Gen. 18611

Kardar M, Parisi G and Zhang Y 1986 Phys. Ret. Lett. 56889

Meakin P 1985 J. Colloid Interface Sci. 105240

Meakin P, Ramanlal P, Sander L and Ball R 1986 Phys. Rev. A 345091

Sutherland D $1966 \mathrm{~J}$. Colloid Interface Sci. 22300

Vold M 1963 J. Colloid Interface Sci. 18684 